\title{
L'aménagement dans une Suisse sans image
}

\section{Chronique d'une timide révision avortée}

En automne 1986, Mme l'ex-Conseillère fédérale E. Kopp décidait de nommer une commission d'experts, chargée d'examiner la loi fédérale sur l'aménagement du territoire afin d'en rendre son application plus rigoureuse et d'en améliorer la mise en œuvre quotidienne.

«Ne pas réinventer l'aménagement du territoire, mais limiter la révision au minimum strictement indispensable pour assurer le respect des principes fondamentaux, notamment celui d'une utilisation judicieuse du sol et d'une occupation rationnelle du territoire», telle était la consigne dont s'inspira le groupe d'experts qui, après d'âpres discussions, déposa ses propositions à la fin de 1988 .

Meilleure délimitation de la zone urbanisée et de la zone agricole, lutte contre la thésaurisation des terrains situés en zone à bâtir, encouragement de la qualité de l'urbanisme, assouplissement des conditions requises pour obtenir un permis de construire dans la zone agricole, obligation d'assurer le suivi de l'occupation du territoire et meilleure protection juridique contre les plans d'affectation insatisfaisants, telles étaient, brièvement résumées, les rubriques sous lesquelles on pouvait ranger les diverses propositions majeures présentées sous forme de modifications de différents articles de la loi existante. Aujourd'hui, cinq ans plus tard, après une procédure de consultation complète, les propositions ont fait presque l'unanimité contre elles et le projet de révision est simplement abandonné par le Conseil fédéral.

Décourageant à certains égards pour les auteurs de propositions, ce résultat est cependant riche en signification notamment sur le caractère fragile des consensus obtenus au sein de groupes de travail où les parties les plus opposées, se trouvent réunies. Les résultats de négociation de ce genre sont tels qu'aucune des parties ne peut les cautionner dans leur ensemble.

L'analyse des prises de position lors de la procédure de consultation permet de classer les adversaires du projet en deux camps fort différents même s'ils se rejoignent sur le rejet des propositions.

Les uns considèrent ces dernières comme trop timides, trop marquées par la «Realpolitik». Certaines mesures, celles tendant à rendre le marché des ter- rains à bâtir plus fluide, sont même tenues pour dérisoires. Les avantages que pourraient fournir une ou deux propositions intéressantes ne justifient pas les risques encourus par un réexamen de la loi dont on pourrait perdre le contrôle lors des discussions au Parlement.

Les autres reprochent aux auteurs du projet d'avoir dépassé le cadre de leur mandat, d'avoir corseté l'extension possible de l'urbanisation, d'avoir subordonné le développement de la construction à de nouvelles conditions restrictives, d'avoir offert de nouvelles voies de recours contre les plans d'affectation, bref d'avoir enrichi sans raison impérative une réglementation déjà suffisamment tracassière.

\section{Veillées d'armes en attendant des jours meilleurs}

Sur la base de ces argumentations, nous avons assisté à une guerre des tranchées durant l'année 1989-1990, préfiguration d'un débat sans issue au Parlement où finalement les adversaires sont tombés d'accord pour recommander l'enterrement du rapport, convaincus que des temps meilleurs pourraient se présenter pour reprendre le combat. Mais de quoi seront faits ces temps meilleurs pour les deux camps opposés?

Pour les uns, c'est la perspective de voir l'individu s'exaspérer devant l'accumulation des contraintes qui s'additionnent les unes aux autres, les obstacles de plus en plus nombreux à l'acquisition de son logement, la difficulté de satisfaire le besoin fondamental qu'est le logement en raison de son coût lié à une raréfaction des terrains à bâtir due au «zoning», sans parler des entraves à la liberté que pourrait constituer une politique combinée des transports.

Pour les autres, l'avenir sera marqué par l'indignation de l'individu face à l'insuffisance des mesures, notamment pour améliorer la qualité de la vie et la nécessité d'amener des correctifs politiques d'une certaine force pour rétablir les dysfonctionnements de l'économie de marché en matière de logement et d'intégrer des mesures de protection du paysage et

Victor Ruffy, Dr., Conseil national, 1054 Morrens/ND 
de l'environnement à l'urbanisation. Certains ont même préconisé l'élaboration d'une loi fédérale sur le logement et l'urbanisme.

Un peu caricaturé, mais tout de même assez près de la réalité, nous avons affaire à l'affrontement de deux courants auxquels nous sommes habitués, les partisans de la souplesse, de la flexibilité, partisans d'une libéralisation face à la gamme infinie des besoins de l'homme, confiants en l'économie de marché pour satisfaire ces derniers et, en face d'eux, les interventionnistes qui sur la base d'une raréfaction grandissante du sol, de l'accaparement des richesses sans cause, veulent rétablir une certaine équité.

Le 700e anniversaire de la Confédération appelle des rétrospectives et il est plus tentant que jamais de prendre un peu de recul pour mettre en perspective la situation contemporaine et tenter une projection tout en gardant présent à l'esprit que les politiques ne se succèdent pas nécessairement les unes aux autres de façon linéaire.

\section{Le sort éphémère des conceptions directrices}

Le premier document national concernant l'aménagement du territoire au sens physique du terme date de décembre 1942. A l'époque «l'aménagement national c'est-à-dire l'utilisation systématique du sol» a une importance capitale, en relation avec le programme des occasions de travail. Après avoir défini l'aménagement national et régional ainsi qu'évoqué les réalisations étrangères, le travail fait le point de la situation dans notre pays montrant que la législation et que les études ne le cèdent en rien à celles de nos voisins. Ce qu'il manque en Suisse c'est une conception générale et son absence explique que la Suisse n'ait pas participé au Congrès de l'union internationale de l'habitation et de l'urbanisme de 1937 à Paris.

Frappé par le peu de place réservée aux faits de société et le poids donné à la politique d'affectation des parcelles, au zonage d'une manière générale, le géographe $\mathrm{E}$. Winkler écrivait: «En premier lieu, le territoire, au sens géographique du terme, est une trame serrée de relations spatio-temporelles unissant sol, climat, eau et organismes. En aucun cas, il n'est réductible à un seul de ces éléments. C'est pourquoi la limitation de l'aménagement du territoire au bien-fonds et au sol va s'avérer être une conception insuffisante du problème.»

Vingt-cinq ans après, fleurirent les conceptions directrices grâce à la loi fédérale sur le logement du 19 mars 1965. Jamais on assista en Suisse à un brassage d'idées aussi complet durant ce siècle. Plein d'espoir, V. Geissberger écrivait en préambule de sa conception consacrée à la "politique nationale», non publiée, «il s'agit de projeter une image de la Suisse vivante de demain». On avait alors foi en une organisation de l'espace idéale, le crédit apporté à l'aménagement du territoire découlait des effets secondaires rapides et destructeurs d'une croissance effrénée. L'abondance des moyens financiers laissait les collectivités publiques sans trop de soucis. En ce temps-la, nombreux étaient ceux qui acceptant les vertus de l'utopie, oubliaient un peu le contexte politique immédiat et pensaient que cette «nouvelle science» devait remédier à tous les maux provoqués par la surchauffe. Certains d'entre eux allaient même jusqu'à penser que l'on allait susciter une société et un monde nouveau. Il y avait la réalité et son contraire, l'image idéale à atteindre à travers des moyens pratiquement hors de portée. Devant une telle tâche, la société était apparemment prête à mettre le prix à se dépasser aussi.

L'évolution de l'histoire récente a voulu que les résultats de travaux et les propositions tombent au moment d'une rupture dans le développement. Alors qu'on discutait des conceptions directrices et les plans directeurs à l'échelle nationale, s'amorçait un mouvement de stagnation sur le plan économique.

Dès 1973, la modification profonde de la conjoncture allait reléguer à l'arrière-plan des préoccupations que l'on pouvait peut-être prendre en considération lors du boom économique, mais qui apparaissaient, dans ces circonstances nouvelles, comme une entrave supplémentaire au développement. C'est dire qu'au moment où l'aménagement du territoire était devenu objet de politique concrète, il cessait de rassembler, pire il devenait impopulaire. Sans jamais prendre la forme d'une récession, l'accalmie économique se chargea de tarir la source des idées anticipatrices devenues suspectes, ou à tout le moins superflues.

Coupé d'un référentiel à large échelle, l'intérêt prédominant pour le concret, le palpable, le matériel, allait refermer l'angle de vue, et l'économisme néolibéral trouvait un champ d'application supplémentaire dans l'aménagement du territoire; en dix ans on était passé de l'espace imaginé à la gestion du sol. La courbe de vie d'une conception séduisante de l'aménagement du territoire de la fin des années 60 n'est pas sans analogie avec celle du projet de nouvelle constitution suisse.

\section{L'absence regrettable d'une image dans la perspective européenne}

Quels que soient les penchants ou, à l'inverse, les sentiments d'aversion que l'on a vis-à-vis des plans dans une société aussi dense que la nôtre, et, compte tenu en plus des moyens matériels dont elle dispose, on doit se demander s'il est bien sage de ne pas avoir de schéma directeur voire de plan directeur à l'échelle nationale. La Suisse, une grande mé- 
tropole en germe sinon déjà en fait, commande de réfléchir à cette échelle sur les composantes majeures de l'espace helvétique, ceci d'autant plus que les cantons sont souvent en difficulté de mettre sur pied des politiques communes pour leurs régions limitrophes. Pourra-t-on vraiment se satisfaire du patchwork résultant de la juxtaposition des plans directeurs cantonaux?

Nous continuons d'opérer de manière indirecte, au coup par coup, secteur par secteur, sans avoir toujours une idée correcte des complémentarités existant entre eux. La conception «Rail 2000» est un des exemples récents les plus frappants.

Dans le contexte contemporain de désillusion et de démobilisation, l'avis est largement partagé que notre comportement politique et notre politique elle-même changeront sous la pression des faits et des processus extérieurs. Est-ce vrai pour notre conception de l'aménagement du territoire? Que nous réserve cette grande libéralisation promise dans le cadre de l'Acte unique et quels enseignements tirer des pratiques de nos grands voisins?

A l'évidence, la mise en place de cet Espace Economique Européen va bouleverser un certain nombre de données. Des axes vont être privilégiés, nous ne le savons que trop, des pôles renforcés, des vastes zones transformées. la société européenne des décideurs, toute fascinée par le prochain coup de fouet donné aux échanges, s'est peu préoccupée des conséquences notamment spatiales de cette ouverture et de cette intensification des mouvements. Un examen rapide de la situation ne nous permet pas d'être très positifs. Les travaux concernant l'élaboration d'un schéma directeur européen piétinent; en fait, une conception de l'espace européen n'est pas allée de pair avec les visions économiques. Les conflits qui ont surgi au sujet de la circulation de transit et les impératifs d'une politique de l'environnement montrent assez les difficultés que nous aurons à faire accepter la subordination de l'économie à l'écologie. On ne sait encore, en ce qui concerne le renforcement des pôles, sur quelle base et dans quelles intentions celui-ci sera opéré. Il semble que nous ne soyons pas sorti d'une politique de rivalité, que les choix font l'objet de négociation, d'où par exemple les préoccupations régionales sont étrangères. Il est étrange que l'Agence européenne de l'environnement, dont on parle depuis deux ans, n'est toujours pas opérationnelle faute de décision sur le lieu de son siège. Enfin en ce qui concerne la gestion des grands espaces, on peut se demander si notamment la politique agricole communautaire est très raisonnable vis-à-vis des impératifs écologiques et des exigences liées à l'entretien du paysage, sans parler ici des structures sociales agraires. Enfin, il faut signaler les dégâts causés par le climat de libéralisation dans la politique d'affectation. Dans certains pays, on a l'impression que la politique des zones a été mise en échec par la pression économi- que. En fait, l'Espace Européen est d'abord économique et les principes de libre circulation des personnes, des produits, des capitaux, des services, relèguent à l'arrière-plan les réflexions et les mesures particulières qu'impliquerait son organisation.

Il n'y a donc pas d'enseignement déterminant à tirer de la politique européenne en aménagement du territoire sinon que dans le cadre d'une intégration, nous aurons à lutter pour défendre nos normes de différentes natures qui seront considérées comme exagérées, pénalisantes, discriminantes.

\section{Les limites de la réduction du champ d'action}

L'absence d'un référentiel spatial, d'une mise en cohérence de nos divers secteurs d'activité s'inscrivant dans l'espace se fera probablement sentir de manière cruelle. Et il faut revenir sur la gestion du sol telle qu'elle s'est instaurée à partir des années 80 , pour indiquer quelles sont ses limites dans le contexte actuel et quelles sont les quelques promesses qu'elle pourrait tenir. L'abandon d'une politique à vaste échelle, celle des conceptions directrices notamment, pour une utilisation mesurée du sol, ce principe désormais dominant respecté à travers des plans d'affectation intégrés ou non à des plans directeurs cantonaux, pouvait évidemment laisser songeurs ceux qui espéraient voir apparaître un instrument parmi d'autres d'intégration politique sous la forme de conceptions directrices.

Pouvait-on néanmoins admettre qu'à certaines conditions cette politique puisse mettre en évidence les caractéristiques particulières du sol qui tiennent à des aspects quantitatifs (les limites de l'espace données une fois pour toutes) et qualitatifs liés à ses multiples fonctions? Ces données ne pouvaient-elles pas constituer les fondements d'une nouvelle relation au sol qui serait non plus fonction du prix que l'on peut en retirer, de la rente foncière, mais bien tributaire d'une nouvelle valeur dans laquelle se trouveraient agrégées des fonctions écologiques? Rien n'indique cependant que nous nous orientons dans cette direction. On ruse avec l'inévitable rareté du sol, on refuse d'entrer en matière sur ses conséquences. Plutôt que de reconnaître au sol sa spécificité de bien non reproductible et de discriminant social le plus manifeste, on dénonce la pratique des aménagistes comme la source arbitraire, capricieuse de la pénurie.

Aujourd'hui comme avant, l'économie foncière commande en grand les orientations de l'aménagement du territoire et la rente foncière reste la préoccupation majeure de ceux qui le font. La concurrence entre les divers secteurs d'activité est telle que l'agriculture, toujours victime de l'urbanisation, souhaiterait beaucoup pouvoir récupérer sur la forêt une part des terres qu'elle doit concéder. Dans de telles circonstances, la dispute autour de l'art. 12 
du nouveau projet de loi sur la forêt, qui autoriserait l'inscription de certains empiétements prévus sur les surfaces forestières dans des plans directeurs, n'a rien de stérile. Même cet espace, considéré en Suisse comme "sacré» depuis bientôt un siècle, en raison de son caractère social et écologique, n'est plus à l'abri de menaces. Mais, l'agriculture n'est pas la seule à chercher des terrains à des prix abordables, la construction elle-même est aujourd'hui en quête de terrains à bâtir dont les prix permettraient d'envisager des logements à loyers accessibles. Au prix où est le terrain, toute parcelle est revendiquée et exploitée jusque dans ses derniers recoins et il devient de plus en plus difficile de défendre la soustraction de certains espaces à toute utilisation pour, par exemple, garantir la conservation d'espaces naturels ou encore en limiter certains usages, pour garantir le maintien de paysages agricoles proches de l'état naturel. Le fait que ces lieux constituent des éléments précieux du patrimoine culturel commun ne justifie plus aux yeux de leurs exploitants des mesures de restriction. L'intimidation par la violence physique a fait tristement son apparition dans notre démocratie à propos de mesures concernant la protection de la nature et du paysage.

La croissance qualitative était le leitmotiv des grandes lignes de la législature qui s'achève cette année. L'aménagement du territoire, en tant que tel, n'aura malheureusement pas été le champ d'élection de la qualité et si le Programme national de recherches consacré au «sol» était parfaitement justifié dans une telle perspective, on doit encore s'interroger sur l'utilisation qui sera faite des résultats. Il est difficile en effet d'être catégorique sur la portée politique d'un tel programme et de dire si les enseignements et les mesures préconisées trouveront des prolongements dans des actions ou si ce programme n'aura été qu'un exercice de style ayant valeur d'alibi pour un pouvoir politique qui prendra ses distances.

Le sentiment prévaut que la progressive dégradation de l'environnement sera malheureusement plus déterminante pour la reconnaissance de nouvelles valeurs que la capacité d'anticipation et d'engagement immédiat de la majorité des acteurs politiques. A l'instar de bien d'autres pratiques commandées par la dynamique, celle de l'aménagement du territoire souffre d'un blocage qui la rend actuellement bien peu séduisante. Ce temps mort contraste douloureusement avec la mobilisation recherchée à travers l'idée d'une Confédération moderne. Il y a 20 ans, l'occasion a été manquée et l'élan brisé; l'invocation de l'utopie ne saurait suffire pour surmonter l'aversion des images des uns et stimuler comme par enchantement l'imagination des autres, ceux qui portent désormais un regard désabusé sur le conservatisme helvétique. 